\title{
Development of a Mixing Mechanism with a Complex Motion of the End-effector
}

\author{
Alexey Fomin* - Wsevolod Ivanov \\ Siberian State Industrial University, Russia
}

This study is focused on the development and analysis of a single-driven mixing mechanism, synthesized with a minimum number of links and joints. The end-effector of this mechanism is designed as a screw ending with a mixing element (blades) moving along a spatial trajectory. The end-effector is set between movable links in the kinematic chain of the mechanism. This allows increasing the working zone of the endeffector. The study provides kinematic analysis via an analytical method of coordinate transformations that have resulted in the detection of variable kinematic parameters (tilt angles and displacements in joints) depending on driving link rotation angle. Based on these results, the motion trajectory of the mixing element has been analytically determined and reproduced. Analytical findings have been verified by results of motion simulations. The presented mechanism is advisable to use for the preparation of various mixtures and levelling the concentrations and temperature of mixed substances. The mechanism can be used in the food, medical, chemical, and construction industries, as well as in agriculture.

Keywords: kinematic pair, mixing mechanism, degree-of-freedom, spatial trajectory, analytical kinematics.

Highlights

- A novel single-driven mixing mechanism in which an end-effector reproduces a movement with four degrees-of-freedom has been synthesized.

- It has been found that the developed mechanism is the simplest one in terms of numbers and types of joints and links for the reproduction of prescribed motion with four degrees-of-freedom.

- Kinematic analysis has been carried out with the definition of all variable parameters (tilt angles and displacements in joints), and the spatial trajectory of the end-effector has been calculated and reproduced.

- The proposed mechanism can be applied for preparation of various mixtures and the levelling of concentrations and temperatures in mixed substances.

\section{INTRODUCTION}

Improvements in the machine-building industry have radically affected the development of associated industries by the introduction of advanced and highperformance techniques. It has become possible to ensure advanced characteristics of created machines and mechanisms as early as at the design stage.

One of the basic stages in the creation of any mechanical system is the structural synthesis (design) of its kinematic scheme, considering the numbers and types of links and joints, as well as their placement in a kinematic chain. One study [1] provides a synthesis method that allows obtaining these parameters for parallel mechanisms based on the theory of linear transformations. Another synthesis method is shown in [2]; it is applied for serial-parallel mechanisms with spatial multi-loop kinematic chains based on the displacement group theory and the Assur principle. In another study [3], the authors show a design method of the five-bar screw-lever manipulator and vary its endeffector's trajectory. The proposed method is based on searching for optimal geometries of links that allow reproducing complex screw trajectories by the end- effector. One study [4] provides fundamental concepts for diverse mechanical systems behind the design, analysis, control, and programming.

Thus, it becomes possible to guarantee the required law motion of a designed system and its high efficiency at the stage of the structural synthesis.

In addition, the provision of the minimum number of drives and movable elements in designed systems is a relevant task, the solution of which allows ensuring advanced reliability, high speed, and efficiency, as well as reducing structural loads in links and joints during its operation. In [5], a systematic procedure for the synthesis of single-loop compliant mechanisms having minimum elements is provided. These mechanisms are applied for adaptive structures. Another study that proposes a synthesis of mechanisms with minimum elements is [6], in which Grubler's method is used for designing mechanisms with two general imposed constraints. In [7], the optimal design of mechanisms considering tolerances on link lengths and clearances in joints is applied for four-bar linkages as the simplest systems. In [8], a multi-objective optimization procedure for a robotic 
arm is developed based on the implementation of design criteria.

The presented study is focused on the synthesis of mixing mechanisms. Fig. 1 shows the simplest mixing mechanisms based on the kinematic schemes of single-driven planar four-bar linkages: crank-rocker (Fig. 1a) and crank-slider (Fig. 1b). Both linkages include fixed link 1 , crank 2, coupler 3, oscillating link 4 , and mixing element 5 . Oscillating link 4 is made as a rocker in Fig. 1a, when it is made as a slide block in Fig. 1 b.

In both mechanisms, the end-effector is coupler 3 that is set between movable links 2 and 4. This feature makes it possible to reach an increased working zone of mixing element 5 , which is rigidly mounted on coupler 3 in both mechanisms. The fault of construction of such mechanisms is that mixing element 5 moves along a planar trajectory. If the spatial motion of the mixing element could be reached, the efficiency of the mixing process would be significantly increased.

There are other mechanisms for mixing operations in which end-effectors are guided along spatial trajectories. There is a mechanism that reproduces complex screw trajectories with a curvilinear axis, presented in [3]. A mixing device that provides a complex trajectory with four dependent motions is shown in [9]. Several parallel mechanisms shown in [10] have been synthesized to reach combined spatial motions by their end-effectors.

Despite the opportunity to reproduce the spatial movements of end-effectors in these mechanisms, the efficiency of their functioning for mixing processes can be improved with the development of their kinematic chains, as well as improvement of output trajectories.
This study aims to develop a novel mixing mechanism, which could include a minimum number of movable links, a single drive, only one-degree-offreedom (DoF) joints, only double-paired links and to provide a movement of the end-effector along a spatial trajectory. Also, the end-effector of the proposed mechanism will be mounted on the intermediate link in the kinematic chain to obtain its increased working zone.

\section{SYNTHESIS OF KINEMATIC SCHEME OF MIXING MECHANISM}

Simple mechanisms, those that have a minimal number of links and joints, are preferable for practical reasons. The design issue of such mechanisms is directly related to finding the ratio between the number of DoF of a designed system, the numbers and types of links and joints, as well as the number of general constraints imposed on a whole system. These parameters are considered in the universal formula of mobility [11], written below:

$$
W_{m}=(6-m) n-\sum_{k-m=1}^{k=5}(k-m) p_{k},
$$

where $W_{m}$ is the mobility of a mechanical system determining the number of its DoF, $m$ is the number of general constraints imposed on the entire mechanical system, $n$ is the number of movable links, $k$ is a class of kinematic pairs (joints) determining their DoF, from 5 to 1. Eq. (1) can be used to calculate structural parameters for synthesis of mechanisms with mobility $W_{m}>0$, or kinematic chains with $W_{m}<0$, or groups of zero mobility (Assur groups) with $W_{m}=0$ [12] and [13]. This formula also allows carrying out structural
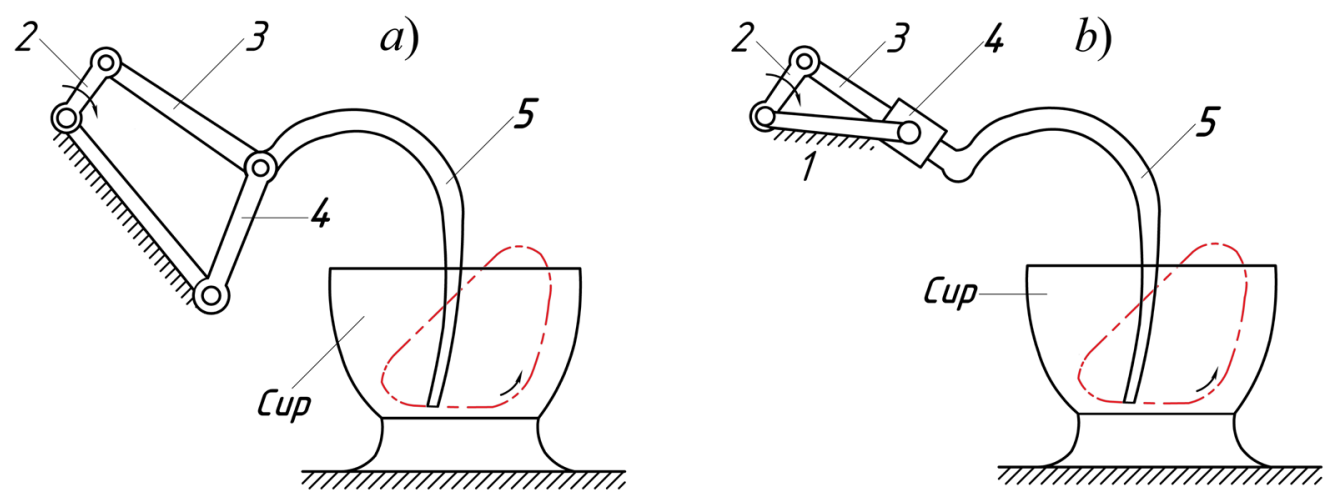

Fig. 1. Kinematic schemes of mixing mechanisms based on planar four-bar linkages: a) crank-rocker, and b) crank-slider 
analysis of mentioned kinematic chains with different mobility.

For the aim of this study, accept the following conditions for the synthesized mechanism: having single drive $\left(W_{m}=1\right)$; provision of one DoF for all joints $\left(p_{5} \neq 0, p_{4}=0, p_{3}=0, p_{2}=0, p_{1}=0\right)$; using only double-paired links (i.e., links that form two joints among themselves); ensuring a spatial motion with four DoF by the end-effector $(m=2)$. According to these conditions, Eq. (1) is transformed into the following form:

$$
n=\left(3 p_{5}+1\right) / 4 .
$$

According to (2), parameter $n$ can take following values $1,4,7,10,13, \ldots$, when $p_{5}$ is equal to $1,5,9$, 13, 17, etc. The first solution when $n=1$ and $p_{5}=1$ describes the simplest mechanism, a crank or a slider. The next solution is when $n=4$ and $p_{5}=5$. According to this solution, the kinematic scheme of the planar mechanism shown in Fig. $1 \mathrm{~b}$ will be transformed into the spatial form. At the same time, the position of all links in one or several parallel planes will be provided to minimize dynamic loads during movement.

The mechanism designed on this idea and included calculated parameters is shown in Fig. 2. This mechanism involves fixed link 1 , crank 2 , coupler 3 , screw 4 , at the end of which mixing element with blades 5 is rigidly mounted, and rocker 6 made in the form of a nut. Kinematic pairs of links 1-2, 2-3, 3-4, and 1-6 are rotational, and pair of links 4-6 is a screw. Moreover, the geometrical axes of joints 1-2, 2-3, and 1-6 are parallel to each other, and the geometrical axes of joints 3-4 and 4-6 lie in the perpendicular plane to them.

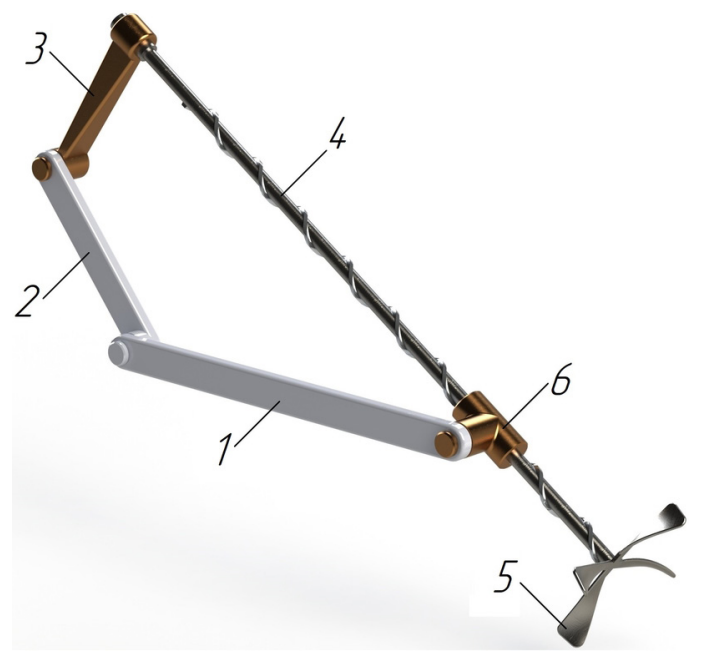

Fig. 2. Mixing mechanism with a complex motion of the end-effector
The input motion in this mechanism is realized by rotation of crank 2 around the geometric axis of links 1-2. According to Eq. (1), the mobility of the mechanism is equal to one $\left(W_{2}=1\right)$. As distinct from the other four DoF mechanisms, such as 3T1R parallel manipulators shown in [14], models of deltarobot shown in [15] and [16], the proposed mixing mechanism has different types of end-effector motions. The end-effector, screw 4 with blades 6 , has three motions (two prismatic and one rotational) in a single plane and additional rotational motion around the axial axis of screw 4.

The operational principle of this mechanism is in the following. When crank 2 is actuated, it drives coupler 3 , which puts in motion screw 4 . The distance between joints 3-4 and 4-6 will be changed, and rocker 6 will incline screw 4 with blades 5 . Due to the presence of screw joint 4-6 and rotational joint 3-4 having an identical spinning axis, screw 4 has additional rotation around its axial axis.

Thus, the developed mechanism has a minimum number of links and single drive, while it realizes a spatial movement of blades 5 with four DoF. A minimum number of links and the presence of only one-DoF joints ensure the simplicity of the mechanism's design and increase the reliability of its functioning.

The application of this mechanism can be associated with performing various operations related to mixing. It can be used in the food, medical, chemical, and construction industries, as well as agriculture as a device for the preparation of homogeneous mixtures, as well as a device providing intensification of mass and heat exchange processes, equalizing the concentration and temperature of mixed substances.

\section{KINEMATIC ANALYSIS OF MIXING MECHANISM}

Regarding the motion trajectory definition of blades 5 , it is appropriate to apply an analytical method of coordinate transformation for the kinematic analysis of the discussed mechanism as blades 5 are directed along a spatial trajectory. This method is widely used in analysing open and closed spatial kinematic chains. In [17], this method is applied to the analysis of the biped robot Pasibot as open kinematic chain. The analysis of closed kinematic chains is presented in [18], in which a 3CPS parallel manipulator is studied, and in [19], in which the mechanism for internal surfaces cutting is investigated. Fig. 3 shows the kinematic scheme of the mixing mechanism, in which spatial coordinate systems are connected with 
each link. The origin of coordinate system $O_{1} x_{1} y_{1} z_{1}$ is connected with link 1 and located in joint 1-2.

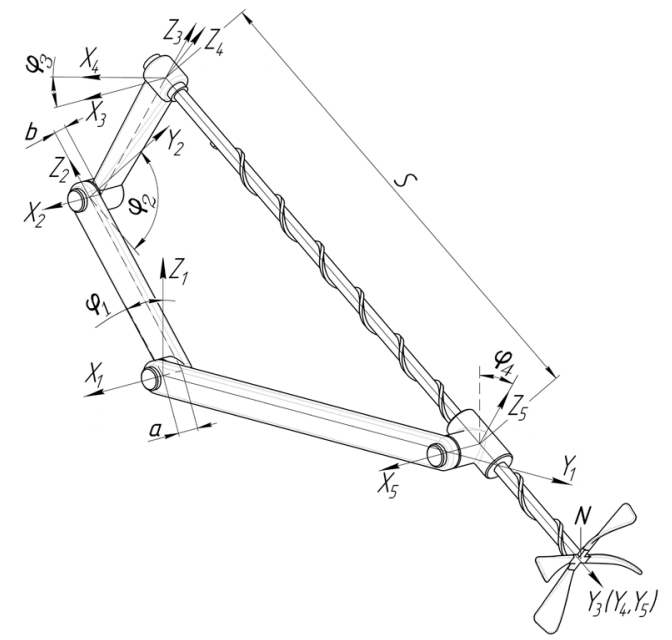

Fig. 3. Toward a kinematic analysis of the mixing mechanism

The origin of the coordinate system $O_{2} x_{2} y_{2} z_{2}$ is connected with crank 2 and located in joint 2-3. Axes $x_{1}$ and $x_{2}$ are parallel to each other. The mechanism is actuated by the rotation of crank 2 around axis $x_{1}$ at angle $\varphi_{1}$, which is a given parameter. The position of coordinate system $O_{2} x_{2} y_{2} z_{2}$ in system $O_{1} x_{1} y_{1} z_{1}$ is determined with the following Denavit and Hartenberg matrix:

$$
\mathbf{T}^{(2,1)}=\left(\begin{array}{cccc}
1 & 0 & 0 & 0 \\
-a & 1 & 0 & 0 \\
-l_{2} \sin \varphi_{1} & 0 & \cos \varphi_{1} & -\sin \varphi_{1} \\
l_{2} \cos \varphi_{1} & 0 & \sin \varphi_{1} & \cos \varphi_{1}
\end{array}\right),
$$

where $a$ is given structural dimension; $l_{2}$ is the length of crank 2. Parameters $a$ and $l_{2}$ define the displacement of coordinate system $O_{2} x_{2} y_{2} z_{2}$ relative to system $O_{1} x_{1} y_{1} z_{1}$.

The origin of the coordinate system $O_{3} x_{3} y_{3} z_{3}$ is connected with coupler 3 and located in joint 3-4. Axes $x_{2}$ and $x_{3}$ are parallel to each other. The position of coordinate system $O_{3} x_{3} y_{3} z_{3}$ in system $O_{2} x_{2} y_{2} z_{2}$ is described using a Denavit and Hartenberg matrix, written in the following form:

$$
\mathbf{T}^{(3,2)}=\left(\begin{array}{cccc}
1 & 0 & 0 & 0 \\
-b & 1 & 0 & 0 \\
-l_{3} \sin \varphi_{2} & 0 & \cos \varphi_{2} & \sin \varphi_{2} \\
l_{3} \cos \varphi_{2} & 0 & -\sin \varphi_{2} & \cos \varphi_{2}
\end{array}\right),
$$

where $b$ is given structural dimension; $l_{3}$ is the length of coupler $3 ; \varphi_{2}$ is a variable parameter describing the rotation of coupler 3 relative to crank 2. Parameters $b$ and $l_{3}$ define the displacement of coordinate system $O_{3} x_{3} y_{3} z_{3}$ relative to system $O_{2} x_{2} y_{2} z_{2}$.

The origin of coordinate system $O_{4} x_{4} y_{4} z_{4}$ is connected with screw 4 and located in joint 3-4, where axes $y_{3}$ and $y_{4}$ are coincident. The position of coordinate system $O_{4} x_{4} y_{4} z_{4}$ in system $O_{3} x_{3} y_{3} z_{3}$ is defined with the following Denavit and Hartenberg matrix:

$$
\mathbf{T}^{(4,3)}=\left(\begin{array}{cccc}
1 & 0 & 0 & 0 \\
0 & \cos \varphi_{3} & 0 & -\sin \varphi_{3} \\
0 & 0 & 1 & 0 \\
0 & \sin \varphi_{3} & 0 & \cos \varphi_{3}
\end{array}\right),
$$

where $\varphi_{3}$ is a variable parameter describing the rotation of screw 4 relative to coupler 3 .

The origin of coordinate system $O_{5} x_{5} y_{5} z_{5}$ is connected with rocker 6 and located in joint $6-1$. The position of coordinate system $O_{5} x_{5} y_{5} z_{5}$ in system $O_{4} x_{4} y_{4} z_{4}$ is described with the following Denavit and Hartenberg matrix:

$$
\mathbf{T}^{(5,4)}=\left(\begin{array}{cccc}
1 & 0 & 0 & 0 \\
0 & \cos \varphi_{3} & 0 & \sin \varphi_{3} \\
S & 0 & 1 & 0 \\
0 & -\sin \varphi_{3} & 0 & \cos \varphi_{3}
\end{array}\right),
$$

where $S$ is a variable parameter describing the distance between joints 3-4 and 4-6. Parameters $S$ and $\varphi_{3}$ are linked through screw pitch $p$, which is a given parameter.

The position of coordinate system $O_{1} x_{1} y_{1} z_{1}$ in system $O_{5} x_{5} y_{5} z_{5}$ is described with the following Denavit and Hartenberg matrix:

$$
\mathbf{T}^{(1,5)}=\left(\begin{array}{cccc}
1 & 0 & 0 & 0 \\
a+b & 1 & 0 & 0 \\
-l_{1} \cos \varphi_{4} & 0 & \cos \varphi_{4} & -\sin \varphi_{4} \\
-l_{1} \sin \varphi_{4} & 0 & \sin \varphi_{4} & \cos \varphi_{4}
\end{array}\right),
$$

where $\varphi_{4}$ is a variable parameter describing the rotation of rocker 6 relative to fixed link $1 ; l_{1}$ is the length of fixed link 1.

The closedness equation of the closed contour of the investigated mechanism is written as:

$$
\mathbf{T}^{(2,1)} \mathbf{T}^{(3,2)} \mathbf{T}^{(4,3)} \mathbf{T}^{(5,4)} \mathbf{T}^{(1,5)}=\mathbf{E},
$$

where $\mathbf{E}$ is identity matrix.

The position of end point $N$ at screw 4 (Fig. 3) in coordinate system $O_{1} x_{1} y_{1} z_{1}$ is defined by radiusvector $\bar{r}_{N}$ from the following equation: 


$$
\bar{r}_{N}=\left(\begin{array}{c}
1 \\
a+b \\
y_{1 N} \\
z_{1 N}
\end{array}\right)=\mathbf{T}^{(2,1)} \mathbf{T}^{(3,2)} \mathbf{T}^{(4,3)}\left(\begin{array}{c}
1 \\
0 \\
l_{4} \\
0
\end{array}\right),
$$

where $y_{1 N}$ and $z_{1 N}$ are coordinates of point $N$ in system $O_{1} x_{1} y_{1} z_{1} ; l_{4}$ is the length of screw 4 . The numerical values of angles $\varphi_{2}, \varphi_{3}, \varphi_{4}$ and displacement $S$ are found from Eq. (8).

\section{RESULTS AND DISCUSSION}

For the numerical calculation, the following parameters have been accepted: $l_{1}=600 \mathrm{~mm}, l_{2}=300$ $\mathrm{mm}, l_{3}=246 \mathrm{~mm}, l_{4}=1040 \mathrm{~mm}, a=40 \mathrm{~mm}, b=25$ $\mathrm{mm}$, and $p=100 \mathrm{~mm}$. Table 1 provides the numerical values of angles $\varphi_{2}, \varphi_{3}, \varphi_{4}$, displacement $S$, coordinates $y_{1 N}, z_{1 N}$ and the length of radius-vector $\bar{r}_{N}$ depending on angle $\varphi_{1}$.

Fig. 4 provides a graphical interpretation of the dependences of angles $\varphi_{2}, \varphi_{3}, \varphi_{4}$ and displacement $S$ on angle $\varphi_{1}$, defining a position of the driving link. These graphics have are in accordance with the analytical values shown in Table 1.

Based on the obtained results, a spatial motion trajectory of blade 5 has been analytically reproduced. It is presented in Fig. 5 relative to base coordinates $O_{1} x_{1} y_{1} z_{1}$ having a centre in joint 1-2. The motion trajectory in Fig. 5 is fundamentally different from

Table 1. Numerical values of variable parameters $\left(\varphi_{2}, \varphi_{3}, \varphi_{4}, S, y_{1 N}, z_{1 N}\right.$ and $\left.r_{1 N}\right)$ depending on angle $\varphi_{1}$ for twelve positions of mixing mechanism

\begin{tabular}{lrrrrrrr}
\hline$\varphi_{1}[\mathrm{deg}]$ & $\varphi_{2}[\mathrm{deg}]$ & $\varphi_{3}[\mathrm{deg}]$ & $\varphi_{4}[\mathrm{deg}]$ & $S[\mathrm{~mm}]$ & $y_{1 N}[\mathrm{~mm}]$ & $z_{1 N}[\mathrm{~mm}]$ & $r_{1 N}[\mathrm{~mm}]$ \\
\hline 0 & 48.06 & 39.07 & 48.06 & 624.16 & 877.29 & -309.33 & 930.23 \\
\hline 30 & 28.24 & -532.05 & 58.24 & 457.79 & 906.50 & -495.00 & 1032.84 \\
\hline 60 & 5.18 & -1191.86 & 65.18 & 278.93 & 919.42 & -690.80 & 1150.02 \\
\hline 90 & -34.97 & -1576.95 & 55.03 & 171.96 & 1097.56 & -711.29 & 1307.89 \\
\hline 120 & -102.40 & -1191.86 & 17.60 & 278.93 & 1325.46 & 230.10 & 1345.28 \\
\hline 150 & -151.76 & -532.04 & -1.76 & 457.79 & 1181.94 & 17.93 & 1182.08 \\
\hline 180 & -185.07 & 39.04 & -5.07 & 624.16 & 1014.22 & 36.73 & 1014.89 \\
\hline 210 & -211.06 & 520.92 & -1.06 & 754.70 & 885.25 & 5.30 & 885.27 \\
\hline 270 & -233.54 & 818.86 & 6.46 & 837.46 & 801.25 & -22.8 & 801.57 \\
\hline 300 & -254.15 & 879.21 & 15.85 & 865.78 & 767.60 & -47.59 & 769.07 \\
\hline 330 & -273.74 & 818.86 & 26.26 & 837.46 & 781.64 & -89.60 & 786.76 \\
\hline & -292.85 & 520.92 & 37.15 & 754.70 & 827.41 & -172.29 & 845.16 \\
\hline
\end{tabular}

a)

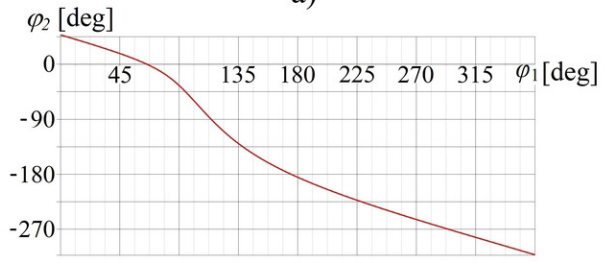

c)

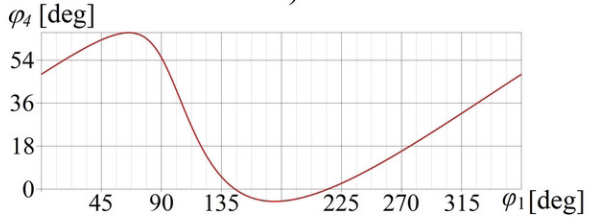

b)

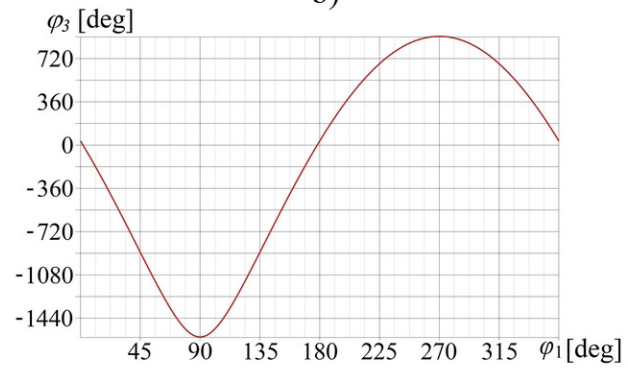

d)

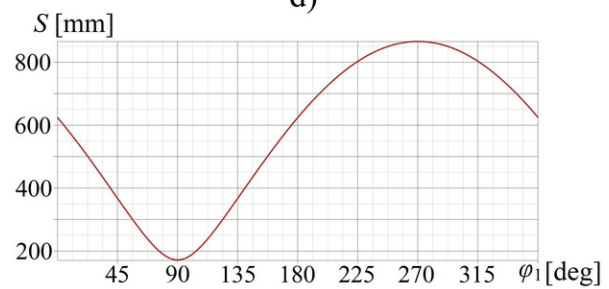

Fig. 4. Graphical interpretation of dependences of variable parameters: a) $\varphi_{2}$, b) $\varphi_{3}$, c) $\varphi_{4}$, and d) $S$ on angle $\varphi_{1}$ 
motion trajectories reproduced by conventional screw mechanisms, proposed in [20] and [21], where the geometric axes of end-effector motions are rectilinear. In the developed mechanism, this axis is made as a closed curvilinear line, the geometry of which is determined by lengths of links $l_{1}, l_{2}, l_{3}$ and $l_{4}$. When changing these parameters, such a trajectory can be varied.

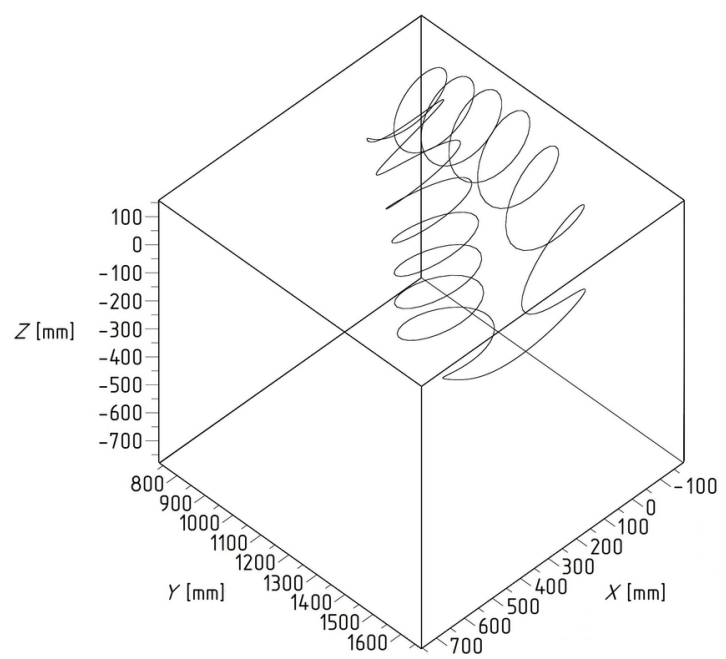

Fig. 5. Analytical motion trajectory of the end-effector that defines the working zone of the mixing mechanism

The results of analytical calculations have been verified using simulation modelling in this study. Specifically, the analytical trajectory of the end- effector presented in Fig. 5 has been reproduced through CAD modelling. Fig. 6 provides a simulated motion trajectory of the mixing mechanism, where the left figure shows the trajectory of the single blade, while the right one shows the trajectories of the four blades. A comparison of analytical and simulated trajectories provides their identity: the simulated trajectory entirely duplicates the analytical one.

\section{CONCLUSIONS}

This study presents a new single-driven mixing mechanism, which includes a minimum number of movable links and joints. To ensure design simplicity and speedwork of this mechanism, all links have been created as double-paired and interconnected only by one-DoF joints. The end-effector is designed as a screw that is set between movable links in the kinematic chain of the mechanism. This placement of the end-effector provides its increased working zone.

Analytical kinematics of the developed mechanism has been solved, and the spatial trajectory of the end-effector that determines its working zone has been reproduced. The trajectory has also been obtained through simulation modelling, which verified analytical calculations. The developed mechanism can be widely applied in various mixing operations and can be used in the food, medical, chemical, and construction industries, as well as in agriculture as a device for creating homogeneous materials and
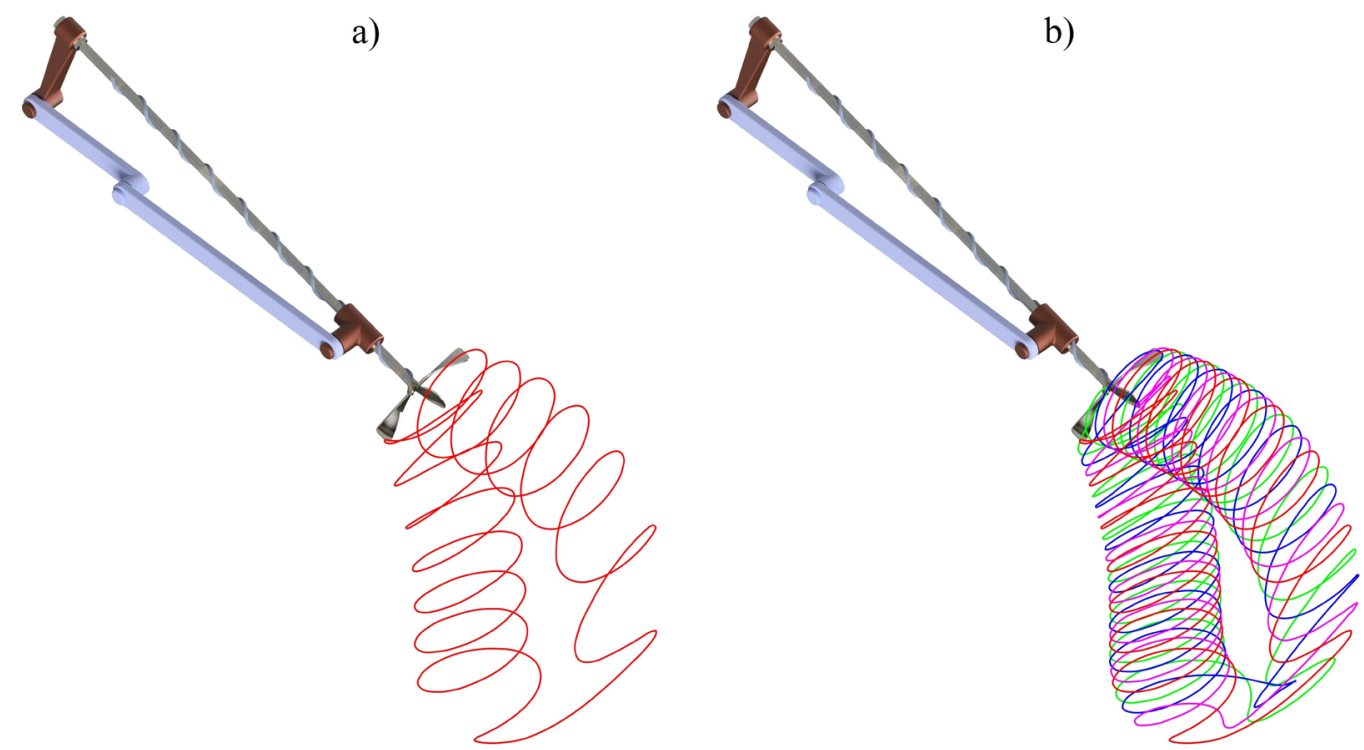

Fig. 6. Simulated motion trajectory of the end-effector that defines the working zone of the mixing mechanism: a) trajectory of the single blade, and b) trajectories of the four blades 
levelling the concentrations and temperature of mixed substances.

\section{ACKNOWLEDGEMENTS}

The reported study was funded by $R F B R$ according to the research project № 18-31-00440.

\section{REFERENCES}

[1] Gogu, G. (2004). Structural synthesis of fully-isotropic translational parallel robots via theory of linear transformations. European Journal of Mechanics - A/ Solids, vol. 23, no. 6, p. 1021-1039, Dol:10.1016/j. euromechsol.2004.08.006.

[2] Zeng, Q., Fang, Y. (2012). Structural synthesis and analysis of serial-parallel hybrid mechanisms with spatial multi-loop kinematic chains. Mechanism and Machine Theory, vol. 49, p. 198-215, D0l:10.1016/j.mechmachtheory.2011.10.008.

[3] Fomin A., Ivanov W., Paik J. (2017). Design of five-bar screwlever manipulator and optimization of its output link path of motion. Procedia Engineering, vol. 206, p. 1703-1708, D01:10.1016/j.proeng.2017.10.701.

[4] Angeles, J. (1987). Fundamentals of Robotic Mechanical Systems: Theory, Methods and Algorithms. Springer, New York, Dol:10.1007/978-3-319-01851-5.

[5] Saggere, L., Kota, S. (2001). Synthesis of planar, compliant four-bar mechanisms for compliant-segment motion generation. Journal of Mechanical Design, vol. 123, no. 4, p. 535-541, Dol:10.1115/1.1416149.

[6] Fomin, A., Paramonov, M. (2016). Synthesis of the four-bar double-constraint mechanisms by the application of the grubler's method. Procedia Engineering, vol. 150, p. 871-877, DOl:10.1016/j.proeng.2016.07.034.

[7] Rhyu, J.H., Kwak, B.M. (1988). Optimal stochastic design of four-bar mechanisms for tolerance and clearance. Journal of Mechanisms, Transmissions, and Automation in Design, vol. 110, no. 3, p. 255-262, Dol:10.1115/1.3267455.

[8] Castejón, C., Carbone, G., García Prada, J.C., Ceccarelli, M. (2010). A multi-objective optimization of a robotic arm for service tasks. Strojniski vestnik - Journal of Mechanical Engineering, vol. 56, no. 5, p. 316-329.

[9] Gileta, V.P., Choosovitin, N.A. (2008). Parametric analysis of device for the mixing with the equal angles overlap and pressure. Proceedings of the Third International Forum on Strategic Technologies, p. 414-416, D0l:10.1109/ IFOST.2008.4602954.
[10] Kheilo, S.V., Glazunov, V.A., Shirinkin, M.A., Kalendarev, A.V. (2013). Possible applications of mechanisms of parallel structure. Journal of Machinery Manufacture and Reliability, vol. 42, no. 5, p. 359-363, D0l:10.3103/S1052618813050063.

[11] Fomin, A., Dvornikov, L., Paramonov, M., Jahr, A. (2012). To the theory of mechanisms subfamilies. IOP Conference Series: Materials Science and Engineering, vol. 124, no. 1, DOI:10.1088/1757-899X/124/1/012055.

[12] Li, S., Dai, J.-S. (2012). Structure synthesis of single-driven metamorphic mechanisms based on the augmented assur groups. Journal of Mechanisms and Robotics, vol. 4, no. 3, 031004, D0I:10.1115/1.4006741.

[13] Dvornikov, L.T. (2008). Towards question about classification of planar Assur groups. Theory of Mechanisms and Machines, vol. 2, no. 6, p. 18-25.

[14] Kong, X., Gosselin, C.M. (2004). Type synthesis of 3T1R 4-DOF parallel manipulators based on screw theory. IEEE Transactions on Robotics and Automation, vol. 20, no. 2, p. 181-190, DOI:10.1109/TRA.2003.820853.

[15] Pierrot, F., Company, O. (1999). H4: a new family of 4-DOF parallel robots. IEEE/ASME International Conference on Advanced Intelligent Mechatronics, p. 508-513, D0I:10.1109/ AIM.1999.803222.

[16] Rey, L., Clavel R. (1999). The Delta Parallel Robot. Boër, C.R., Molinari-Tosatti, L., Smith, K.S. (eds), Parallel Kinematic Machines. Advanced Manufacturing. Springer, London, p. 401-417, DOI:10.1007/978-1-4471-0885-6_29.

[17] Meneses, J., Castejón, C., Corral, E., Rubio, H., García-Prada, H.C. (2011). Kinematics and dynamics of the quasi-passive biped "PASIBOT". Strojniški vestnik - Journal of Mechanical Engineering, vol. 57, no. 12, p. 879-887, D0l:10.5545/svjme.2010.210.

[18] Cheng, G., Xu, P., Yang, D., Li, H., Liu, H. (2013). Analysing kinematics of a novel 3CPS parallel manipulator based on rodrigues parameters. Strojniški vestnik - Journal of Mechanical Engineering, vol. 59, no. 5, p. 291-300, DOl:10.5545/sv-jme.2012.727.

[19] Fomin, A., Paramonov, M. (2019). Structural and kinematic analysis of internal surfaces cutting. Journal of Machinery Manufacture and Reliability, vol. 4, p. 14-21, D0l:10.1134/ S0235711919040072.

[20] Jones, M.H., Velinsky, S.A. (2012). Kinematics of roller migration in the planetary roller screw mechanism. Journal of Mechanical Design, vol. 134, no. 6, 061006 , DOI:10.1115/1.4006529.

[21] Wei, C.C., Lin, J.F. (2004). Kinematic analysis of the ball screw mechanism considering variable contact angles and elastic deformations. Journal of Mechanical Design, vol. 125, no. 4, p. 717-733, DOI:10.1115/1.1623761. 